\title{
Neural differentiation of mouse embryonic stem cells grown in monolayer
}

\author{
Jiří PACHERNÍK ${ }^{\mathrm{a}, \mathrm{c}}$, Milan EŠNER $^{\mathrm{c}}$, Vítězslav BRYJA ${ }^{\mathrm{a}, \mathrm{b}}$, \\ Petr DvořÁK ${ }^{\mathrm{a}, \mathrm{b}, \mathrm{c}}$, Aleš HAMPL ${ }^{\mathrm{a}, \mathrm{b}, \mathrm{c} * *}$ \\ ${ }^{a}$ Center for Cell Therapy and Tissue Repair, Charles University, V úvalu 84, \\ 15006 Prague 5, Czech Republic \\ ${ }^{b}$ Department of Molecular Embryology, Institute of Experimental Medicine, \\ Academy of Sciences of the Czech Republic, Vídeňská 1083, 14220 Prague, Czech Republic \\ ${ }^{c}$ Laboratory of Molecular Embryology, Mendel University Brno, Zemědělská 1, \\ 61300 Brno, Czech Republic
}

(Received 26 February 2002; accepted 24 June 2002)

\begin{abstract}
To drive neural differentiation of mouse embryonic stem (ES) cells, various culture protocols have been previously developed that all require the formation of embryoid bodies, usually combined with a treatment by all-trans retinoic acid (aRA). Here, we investigated whether or not neural differentiation can also occur in a simplified monolayer culture. Mouse ES cells were plated in serum-containing DMEM media with and without aRA and grown under these conditions for 2 days. Then, the cells were transferred to fresh serum-containing DMEM media and/or to serum-free DMEM/F12 media supplemented with a mixture of insulin, transferrin, selenium, and fibronectin (ITSF) for further culture. The changes in cell morphology and in the expression of selected molecular markers were monitored. Generally, in contrast to all the others, the protocol consisting of a 2-day culture in serum-containing DMEM followed by continuous exposure to the ITSF supplement in DMEM/F12 drove a vast majority of ES cells to generate phenotypic signs of neural lineage. Altogether, neural differentiation can be achieved in vitro without the step involving the formation of embryoid bodies as well as the treatment by aRA.
\end{abstract}

embryonic stem cells / neural differentiation

\footnotetext{
* Correspondence and reprints

E-mail: hampl@mendelu.cz
} 


\section{INTRODUCTION}

Mouse embryonic stem cells (ES cells) are immortal cell lines derived from an inner cell mass of the 3.5 day blastocyst that can be propagated in vitro in a nondifferentiated state in the presence of the leukemia inhibitory factor (LIF). When reintroduced back into the mouse blastocyst, they can give rise to cells of any cell lineage of the developing mouse, including the germ line. Importantly, ES cells are also able to generate differentiated progeny in vitro $[6,10$, 15]. This implies that cultured ES cells can both generate and respond to signals that normally regulate murine development. Under specific culture conditions, ES cells form three-dimensional structures called embryoid bodies, from which they spontaneously differentiate into a variety of cell types [19]. In the past few years, strategies have been developed that allow to differentiate ES cells into specific cell lineages, including hematopoietic [24], myogenic [25], neural/neuronal [1, 12, 20], and others.

In order to be driven into a neural lineage, ES cells are usually subjected to a two-step differentiation procedure. First, ES cells are allowed to form embryoid bodies (EBs) by culturing either on nonadherent (bacteriological) dishes or in hanging drops of media. Both media with $[1,12]$ and without all-trans retinoic acid (aRA) [20] can be used. Resulting EBs are then plated on tissue culture dishes and further cultured either in DMEM media containing serum but not LIF and aRA [1, 12] or in a serum-free DMEM/ F12 media supplemented with insulin, transferrin, selenium and fibronectin [20]. These manipulations give rise to cell populations rich in neural and neuronal cells that can be further specialized by treating them with various cytokines such as EGF, FGF-2, and/or PDGF [4].

Although the techniques summarized above are sufficient to give rise to neural progenitor cells and neurons, these cells are produced by selecting out non-neural cell types present in EBs, rather than by homogenous differentiation towards solely neural lineages. Considering that, in terms of cellto-cell interactions, one-dimensional culture introduces lower complexity to the system compared to three-dimensional EBs, we reasoned that differentiation protocols involving only monolayer culture must be developed in order to: (1) make large scale production of neurodifferentiated cells more effective and to (2) allow for analyzing the molecular mechanisms that drive early events of neural differentiation in vitro. Therefore, in this study several protocols strictly involving only adherent monolayer culture were evaluated for their ability to induce neural differentiation of mouse ES cells.

\section{MATERIALS AND METHODS}

\subsection{ES cell culture and experimental design}

Embryonic stem cells, lines D3, C3H, and $\mathrm{F} 1[6,8,14]$, were routinely grown on mitomycin C-treated STO fibroblasts in Dulbecco's modified Eagle medium (DMEM, supplemented with $20 \%$ fetal calf serum, $100 \mathrm{mM}$ nucleosides, $0.05 \mathrm{mM}$ $\beta$-mercaptoethanol, $100 \mathrm{i} . \mathrm{u} \cdot \mathrm{mL}^{-1}$ penicillin, $0.1 \mathrm{mg} \cdot \mathrm{mL}^{-1}$ streptomycin) with 1000 units. $\mathrm{mL}^{-1}$ LIF (GIBCO BRL, CHEMOS CZ, Prague, Czech Republic). For the experiments, STO fibroblasts were removed by centrifugation on a discontinuous FicollPaque gradient (Amersham Pharmacia Biotech, Buckinghamshire, England, 85\% $\mathrm{v} / \mathrm{v}$ in phosphate buffered saline - PBS, $\mathrm{pH}$ 7.4) followed by 45 minutes of adhering on the tissue culture dish. Embryonic stem cells ( $>95 \%$ purity) were plated $(\sim 5 \times$ $10^{3}$ cells per $\mathrm{cm}^{2}$ ) on gelatinized tissue culture dishes into DMEM with and without $0.5 \mu \mathrm{M}$ aRA (Sigma, Prague, Czech Republic). After 48 hours of culture, the media was replaced by either fresh DMEM or ITSF 


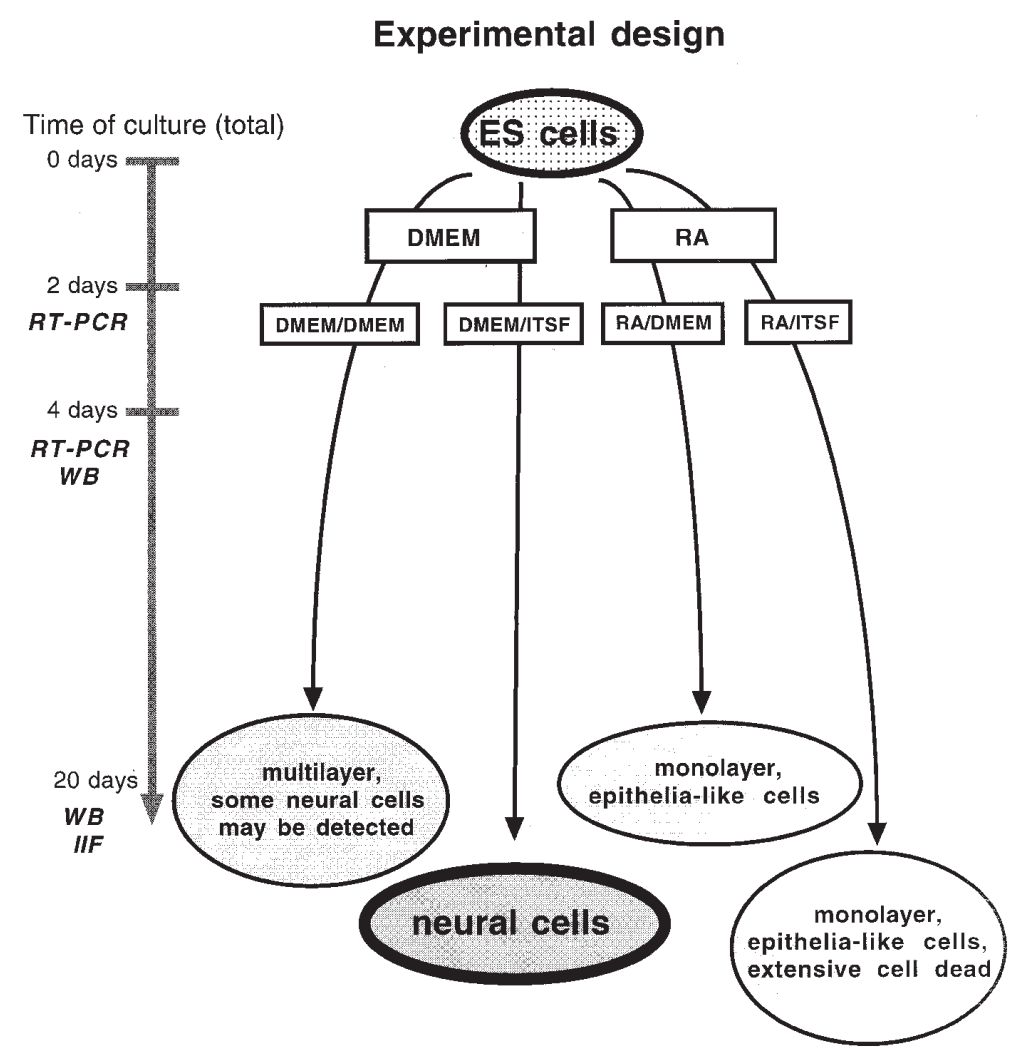

Figure 1. Experimental design; RT-PCR - reverse transcriptase-polymerase chain reaction, WB Western blotting, IIF - indirect immunofluorescence.

media (DMEM/F12 1:1 supplemented with the mixture of transferrin, insulin, selenium (ITS supplement, Sigma) and $1 \mu \mathrm{g} \cdot \mathrm{mL}^{-1}$ fibronectin (GIBCO BRL)). The complete experimental design is shown in Figure 1.

\subsection{Semiquantitative RT-PCR analysis}

Total RNA was extracted from each cell preparation by the RNeasy Mini Kit (QIAGENE, Bio-Consult, Prague, Czech Republic). Complementary DNA was synthesized according to the manufacturer's instructions for M-MLV reverse transcriptase (GIBCO BRL). Primers and PCR conditions were as follows (primer sequence/annealing temperature/cycles/product size): $\mathrm{Pax}-6$
(5'-TGCCCTTCCATCTTTGCTTG-3', 5'-TCTGCCCGTTCAACATCCTTAG3'/54 ${ }^{\circ} \mathrm{C} / 33 / 178$ bp); Brachyury (5'-GAGAGAGAGCGAGCCTCCAAAC-3', 5'-GCTGTGACTGCCTACCAGAATG-3'/ $56^{\circ} \mathrm{C} / 29 / 230 \mathrm{bp}$ ); $\alpha$-fetoprotein (5'-ATGTATGCCCCAGCCATTCTGTCC-3', 5' - GA GA T A A GCC T T C A GG T T TGACGC-3'/54 ${ }^{\circ} \mathrm{C} / 29 / 466$ bp); HPRT (5'-CTTGCTGGTGAAAAGGACCTCTC-3', 5'-CAAATCAAAAGTCTGGGGACGC-3' $56{ }^{\circ} \mathrm{C} / 24 / 350 \mathrm{bp}$ ). Before being used for cDNA quantification, the range of linearity was determined for each primer set. PCR products were separated on $1.5 \%$ agarose gels and visualized by ethidium bromide staining. 


\subsection{Western blotting (WB)}

The following neural markers were quantified by Western blotting: neural cell-adhesion molecule (N-CAM) [18], neuron-specific class III $\beta$-tubulin isotype [7], and growth-associated protein-43 (GAP-43) [21]. The cells were washed with PBS and lysed in sodium dodecyl sulphate (SDS)lysis buffer (50 mM Tris-HCl, pH 7.5; $1 \%$ SDS; $10 \%$ glycerol). Protein concentrations were determined using the DC Protein assay kit (Bio-Rad). Lysates were supplemented with bromophenol blue $(0.01 \%)$ and $\beta$-mercaptoethanol (1\%), and equal amounts of total protein $(10 \mu \mathrm{g})$ were subjected to $10 \%$ SDS-PAGE. After being electrotransferred onto polyvinylidene difluoride membrane (Immobilon-P, Sigma), the proteins were immunodetected using the appropriate primary and secondary antibodies, and visualized by ECL+Plus reagent (Amersham Pharmacia Biotech) according to the manufacturer's instructions. The following primary antibodies were employed: mouse monoclonal antibody against human N-CAM, which cross-reacts with the mouse homolog (C0678; Sigma), mouse monoclonal antibody against neuron-specific class III $\beta$-tubulin isotype (TU-20, gift from Dr. Draber, Institute of Molecular Genetics, Prague, Czech Republic), and the goat polyclonal antibody against human GAP-43, which cross-reacts with the mouse homolog (sc-7457; Santa Cruz Biotechnology, Santa Cruz, CA).

\subsection{Indirect immunofluorescence (IIF)}

The expression of two neural markers, glial fibrillary acidic protein (GFAP) [3] and microtubule-associated protein-2 (MAP-2) [22], was determined by indirect immunofluorescence (IIF) using the following antibodies: goat polyclonal antibody against human GFAP, which cross-reacts with the mouse homolog (sc-6170, Santa Cruz
Biotechnology) and monoclonal antibody against rat MAP-2, which cross-reacts with the mouse homolog (M4403, Sigma). For IIF, ES cells differentiating for 18 days were transferred onto glass coverslips, cultured for another 2 days, fixed in ethanol/acetic acid $(95 \% \mathrm{EtOH}, 1 \%$ acetic acid) for $30 \mathrm{~min}$ on ice, then were slowly rehydrated, quenched with $1 \% \mathrm{BSA}$ in PBS for $1 \mathrm{~h}$ at room temperature, incubated with the appropriate primary (overnight at $4{ }^{\circ} \mathrm{C}$ ) and FITCconjugated secondary (1 hour at RT) antibodies, and mounted to Mowiol (Sigma). The cells were viewed under an epifluorescent microscope Olympus BX-60 (Olympus, Prague, Czech Republic).

\section{RESULTS}

In embryoid bodies, neurodifferentiation normally occurs irrespectively of the presence or absence of aRA in culture media. Still, in order to investigate the capacity of ES cells to produce cells with neural phenotypes under adherent growth conditions, both aRA+ and aRA- cultures (here referred to as RA and DMEM) were set in DMEM for the initial two days, thus timely corresponding to the classical EB-based approach (Fig. 1). After this period, each culture was transferred back to DMEM and/or ITSF media and it was maintained under these conditions for another 18 days. Altogether, the four independent differentiation protocols, further referred to as DMEM/DMEM, DMEM/ITSF, RA/DMEM, and RA/ITSF, were assayed in this study. To monitor both early and late changes occurring in differentiating ES cells, RNA/protein/cell samples were taken at three time points $(2,4$, and 20 days) as depicted in Figure 1.

\subsection{Lineage-specific gene markers}

The levels of mRNAs of the genes that are specific for the ectoderm/neuroectoderm (Pax-6), mesoderm (Brachyury), 


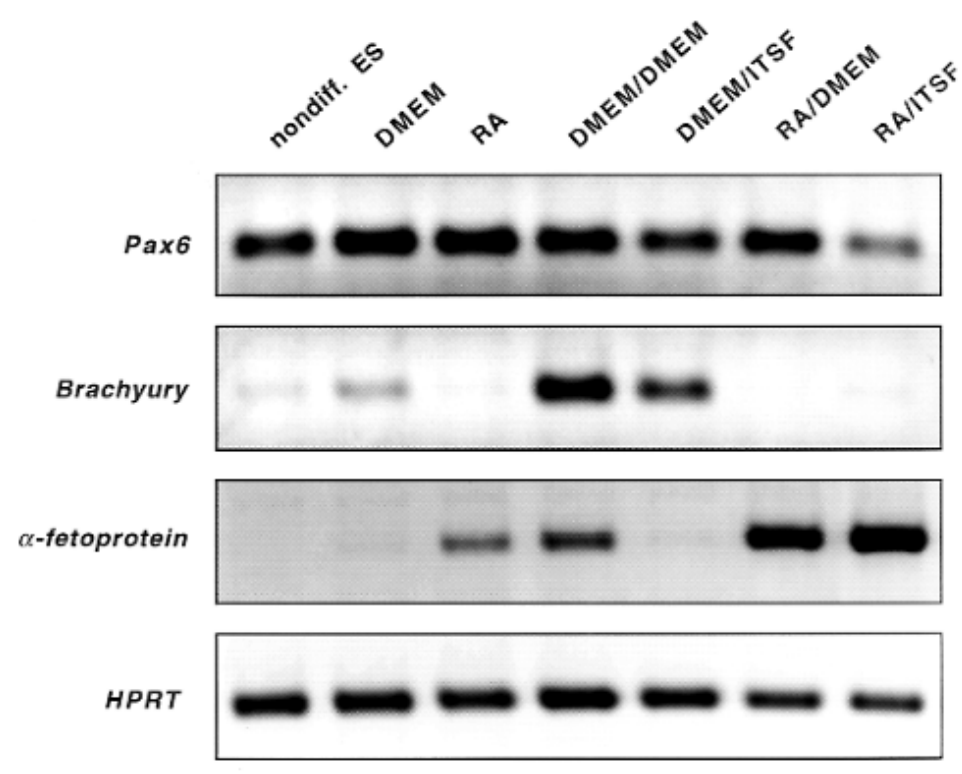

Figure 2. Expression of mRNAs of cell lineage-specific genes in differentiating ES cells (RT-PCR). Embryonic stem cells were first grown for 2 days in the DMEM media with (RA) or without (DMEM) aRA, and then they were cultured for another 2 days in either the DMEM media (RA/DMEM, DMEM/DMEM) or the ITSF media (RA/ITSF, DMEM/ITSF). Data are representative of at least three independent replicates.

and endoderm ( $\alpha$-fetoprotein) were determined by semiquantitative RT-PCR at days 2 and 4 of ES cell differentiation [9, 13, 23]. From all three messages analyzed, only Pax-6 mRNA was found in large amounts in nondifferentiated ES cells (Fig. 2). At 2 days after withdrawal of the LIF and feeder cells (the beginning of the differentiation protocol), differential expression of Brachyury and $\alpha$-fetoprotein developed based on the protocol used. Specifically, aRA-free DMEM media (DMEM) supported the expression of Brachyury while aRA-containing DMEM (RA) promoted the expression of $\alpha$-fetoprotein (Fig. 2). As expected, 2 more days of culture (DMEM/DMEM, DMEM/ITSF, RA/DMEM, and RA/ITSF protocols) brought up further differences in the expression of the marker genes (Fig. 2). While a continuous culture in serumcontaining DMEM (the DMEM/DMEM protocol) has led to the further accumula- tion of the Brachyury message, a simple switch to ITSF (the DMEM/ITSF protocol) diminished this tendency. The presence of aRA in media (the RA/DMEM, and RA/ITSF protocols) thoroughly prevented the occurrence of Brachyury mRNA. The level of $\alpha$-fetoprotein in ES cells cultured in aRA-containing media became strongly upregulated irrespectively of its precise formulation (DMEM and ITSF). On the contrary, media formulation caused a major difference in $\alpha$-fetoprotein expression under aRA-free conditions. Specifically, although DMEM media allowed for the expression of $\alpha$-fetoprotein, this was shut down in ITSF. In contrast to Brachyury and $\alpha$-fetoprotein, all four protocols supported the continuous transcription of Pax-6, with only a slight downregulation being observable at day 4 in the ITSF media (the DMEM/ITSF and RA/ITSF protocols). 


\subsection{Cell morphology}

Along with changes in the expression of gene markers, the morphology of cells also developed specificities according to the particular differentiation protocol. The cells were monitored daily and their morphology, as it was typically observed at days 4 and 20, is shown in Figure 3. First, there was a noticeable difference between the cells that were (the RA/DMEM and RA/ITSF protocols) and that were not (the DMEM/DMEM and DMEM/ITSF protocols) exposed to aRA, irrespectively of the exact media formulation. Generally, treatment with aRA caused the ES cells to strictly grow in a monolayer, while aRA-free conditions allowed for the formation of multilayer structures. Moreover, after about ten days, a massive cell dying took place in the RA/ITSF culture, leaving only a few cells attached to the dish at day 20. Still, the major finding represents the behavior of ES cells that were cultured according to the DMEM/ITSF protocol, combining two days in aRA-free DMEM followed by 18 days in ITSF. These cells continuously slowed their multiplication, rounded up, and started to create protrusions (Fig. 3, Day 4). Although a majority of such cells died, the remaining cells gave rise to three-dimensional structures. Later on, these structures developed into growing spheroids/colonies with expanding neuron-like cells (Fig. 3, Day 20). Such colonies and expanding neurons represented from between 60 to $90 \%$ of all cells in culture depending on the ES cell line used. The highest percentage was produced by F1 ES cells, while the lowest by D3 ES cells.

\subsection{Neural-specific protein markers}

Both Western blot analysis (N-CAM neuron/glia-specific, neuron-specific class III $\beta$-tubulin isotype - neuron-specific, GAP-43 - neuron/glia-specific) and indirect immunofluorescence (GFAP - astro- cyte-specific, MAP-2 - neuron-specific) were employed to determine whether or not the cells with the neural phenotype developed in culture. When Western analyzed at day 4 of culture, no neural marker was detectable under any culture protocol (Fig. 4, Day 4). In contrast, at day 20, correspondingly with their morphology and gene expression profile, aRA-nontreated cells grown in ITSF (protocol DMEM/ITSF) contained high levels of all neural markers analyzed by both WB and IIF (Fig. 4, Day 20; Fig. 5). According to GFAP expression determined by IIF, astrocytes represented about $2 \%$ of the cells under such culture conditions. Notably, some GAP-43-positive cells obviously also developed in aRAfree DMEM cultures (the DMEM/DMEM protocol) (Fig. 4, Day 20, longer exposure). Importantly, the RA/DMEM and RA/ITSF protocols involving aRA-treatment produced no cells positive for any neural marker even upon 20 days of culture.

\section{DISCUSSION}

In this study, mouse ES cells were successfully driven to neurodifferentiate under culture conditions not involving the formation of embryoid bodies and the treatment by all-trans retinoic acid. In other words, simple culturing of dish-adhered mouse ES cells in serum-free DMEM/F12 media supplemented by insulin, transferrin, selenium, and fibronectin can give rise to a highly enriched population of neural cells. Such media was originally shown to be well suited for serum-free culture of rat neuroblastoma cells [2] and then also for neurodifferentiation of mouse embryonal carcinoma cells [5]. When adopted in this study to mouse ES cells, this strategy promoted the elimination of cells expressing nonectodermal gene markers (Fig. 2). Instead, most cells with neural morphological and biochemical features survived and outgrew in such a culture. The resulting spherical colonies were very reminiscent to those seen in 

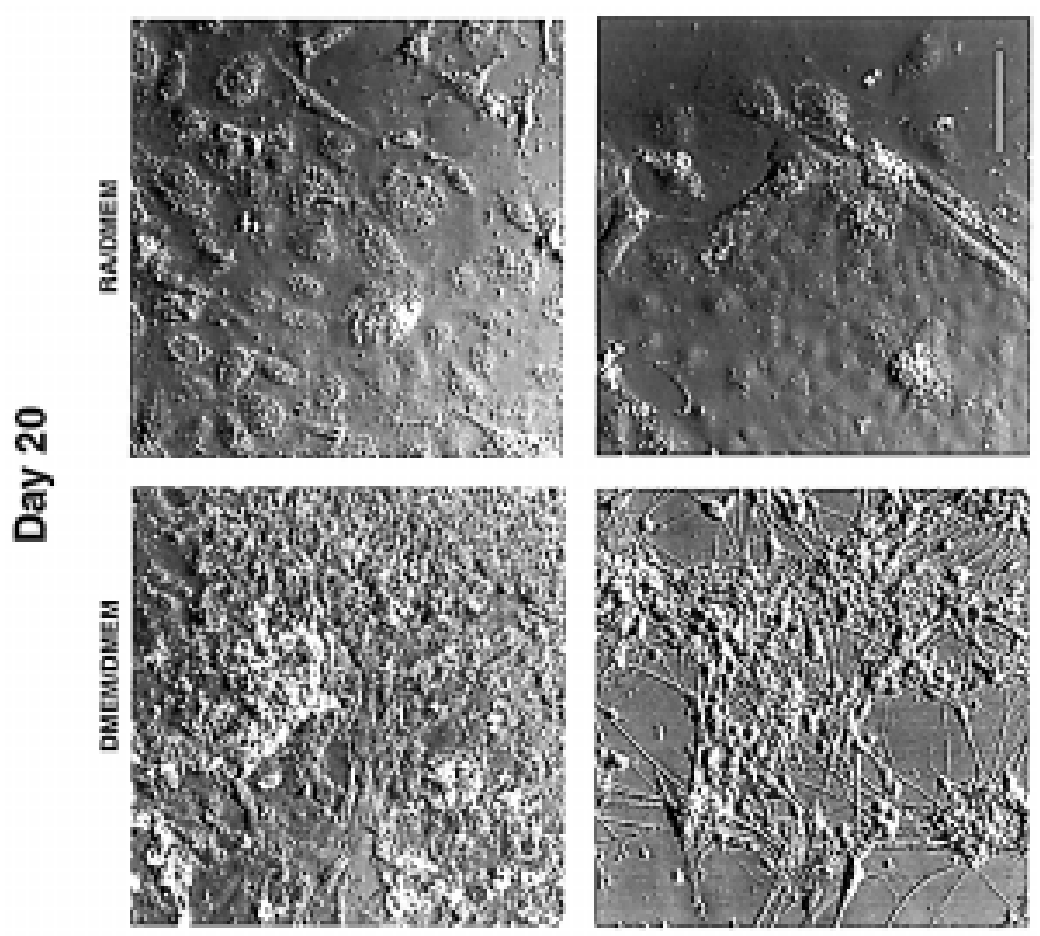

善焉㐫

赵事

잉

$\frac{1}{5}$

है

记

옹.

政密

요음

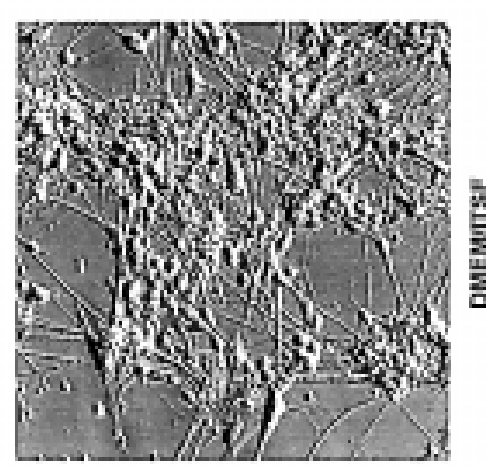

斊壱

ํㅡㄹ

尌安

흥 정

部

政

o

在

尊势

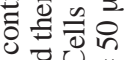

政
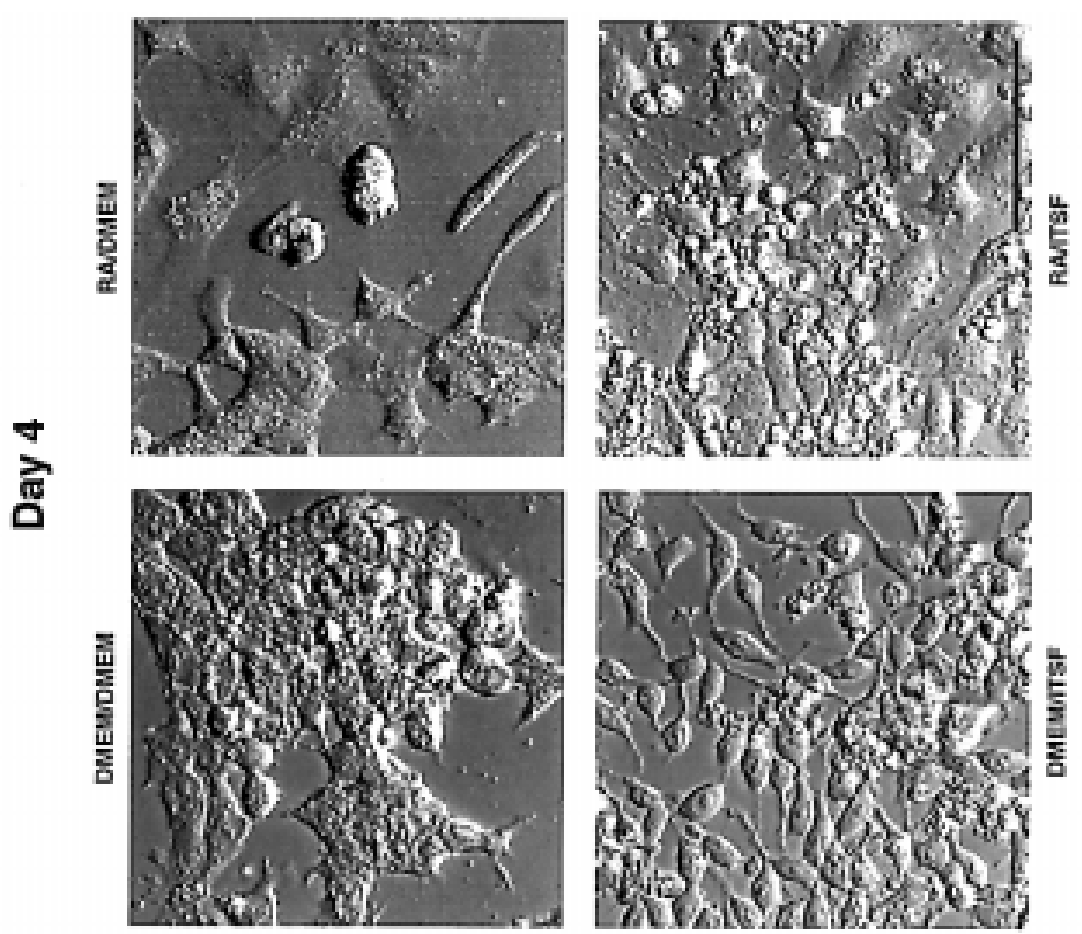

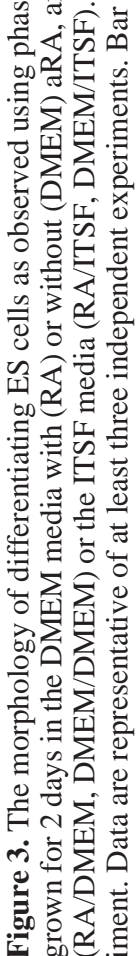



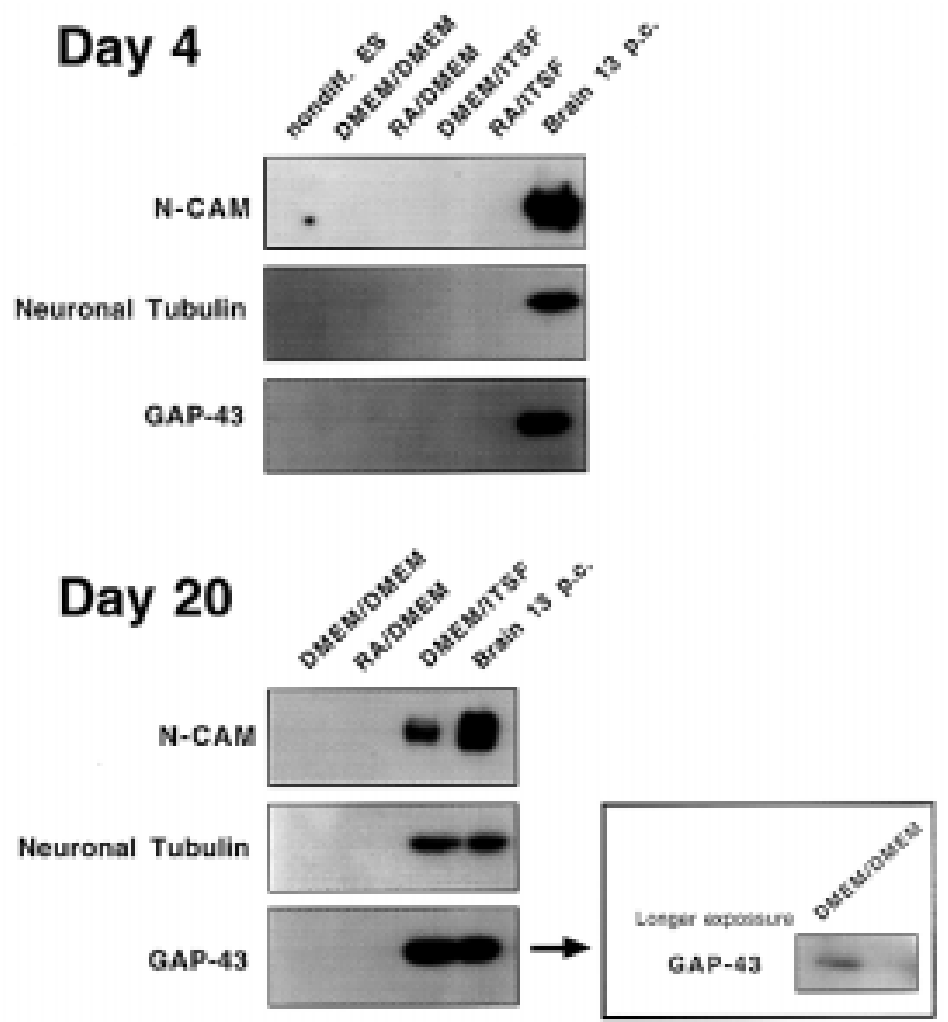

Figure 4. Expression of N-CAM, neuron-specific class III $\beta$-tubulin isotype (neuronal tubulin), and GAP-43 in differentiating ES cells as detected by Western blot. Embryonic stem cells were first grown for 2 days in the DMEM media with (RA) or without (DMEM) aRA, and then they were cultured for another 18 days in either the DMEM media (RA/DMEM, DMEM/DMEM) or the ITSF media (RA/ITSF, DMEM/ITSF). Cells for the analysis were taken at day 4 (Day 4) and at day 20 (Day 20) of the experiment. Equal amounts of total cellular proteins were loaded onto the gel. At day 20 of the RA/ITSF protocol, no cell sample was obtained due to massive cell death. Longer exposure was also shown for day 20 to document some expression of GAP-43 in the cells cultured in DMEM. Data are representative of at least three independent experiments.

GFAP

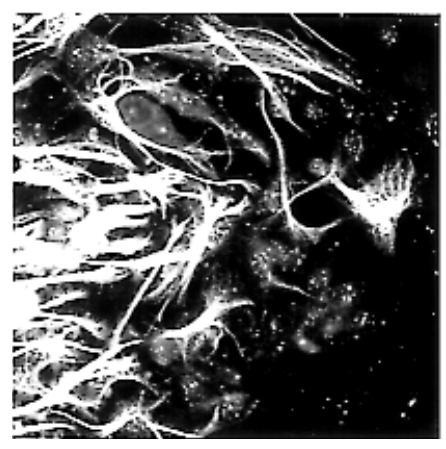

MAP-2

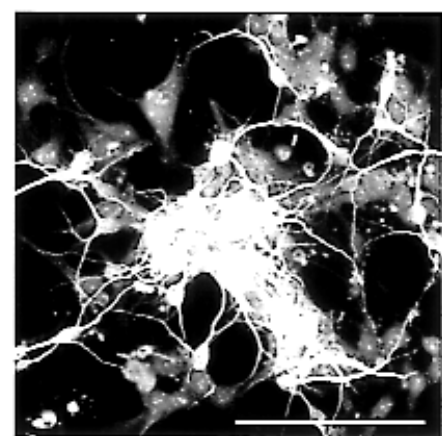

Figure 5. Immunocytochemical detection of GFAP and MAP-2 in differentiating ES cells. Only positively reacting cells cultured for 2 days in DMEM and then 18 days in ITSF are shown (protocol DMEM/ITSF). Data are representative of at least three independent experiments. $\mathrm{Bar}=50 \mu \mathrm{m}$. 
cultured neural stem cells derived from fetal and/or adult human brains [11]. Although treating ES cells derived from embryoid bodies by aRA is beneficial for neurodifferentiation [1, 12], obviously this was not the case for ES cells cultured here under adherent conditions. Correspondingly with what was previously shown by Mummery and colleagues [17] for both ES and embryonal carcinoma cells, in our study, aRA also caused ES cells to develop primarily towards endodermal lineage, as documented by the heavy expression of $\alpha$-fetoprotein mRNA. Retinoic acid was previously shown to induce expression of growth factor receptors in various cell types. Such an increased expression then makes cells more responsive to differentiation-inducing signals, but also more vulnerable to the absence of growth factors [16]. Such a scenario also seems to apply to aRA-treated ES cells, since massive cell death occurred when such cells were cultured here under serum-free conditions (protocol RA/ITSF).

Altogether, the differentiation protocol involving 2 days of culture in a serum-containing media followed by culture in media with only insulin, transferrin, selenium, and fibronectin, was found to preferentially support the development of neural cells from mouse ES cells. This differentiation approach may thus represent a novel alternative to the widely used protocols that invariably employ the formation of embryoid bodies $[1,20]$. It is believed that it might serve as the experimental system for studying the molecular mechanisms involved in neurodifferentiation and also as a basis for the further simplification of generating neural cells in vitro.

\section{ACKNOWLEDGMENTS}

This research was supported in part by the Academy of Sciences of the Czech Republic (AV 0Z5039906), and by the Ministry of Education, Youth, and Sports (MSM 432100001, LN 00A065).

\section{REFERENCES}

[1] Bain G., Kitchens D., Yao M., Huettner J.E., Gottlieg D.I., Embryonic stem cells express neuronal properties in vitro, Dev. Biol. 168 (1995) 342-357.

[2] Bottenstein J.E., Sato G.H., Growth of rat neuroblastoma cell line in serum-free supplemented medium, Proc. Natl. Acad. Sci. USA 76 (1979) 514-517.

[3] Brenner M., Structure and transcriptional regulation of the GFAP gene, Brain Pathol. 4 (1994) 229-237.

[4] Brustle O., Jones K.N., Learish R.D., Karram K., Choudhary K., Wiestler O.D., Duncan I.D. McKay R.D.G., Embryonic stem cell-derived glial precursors: A source of myelinating transplantants, Science 285 (1999) 754-756.

[5] Darmon M., Bottenstein J., Sato G., Neural differentiation following culture of embryonal carcinoma cells in a serum-free defined medium, Dev. Biol. 85 (1981) 463-473.

[6] Doetschman T.C., Eistetter H., Katz M., Schmidt W., Kemler R., The in vitro development of blastocyst-derived embryonic stem cell lines: formation of visceral yolk sac, blood islands and myocardium, J. Embryol. Exp. Morphol. 87 (1985) 27-45.

[7] Draberova E., Lukas Z., Ivanyi D., Viklicky V., Draber P., Expression of class III $\beta$-tubulin in normal and neoplastic human tissues, Histochem. Cell Biol. 109 (1998) 231-239.

[8] Dvorak P., Hampl A., Jirmanova L., Pacholikova J., Kusakabe M., Embryoglycan ectodomains regulate biological activity of FGF-2 to embryonic stem cells, J. Cell Sci. 111 (1998) 2945-2952.

[9] Dziadek M., Adamson E., Localization and synthesis of alphafoetoprotein in post-implantation mouse embryos, J. Embryol. Exp. Morphol. 43 (1978) 289-313.

[10] Evans M.J., Kaufman M.H., Establishment in culture of pluripotential cells from mouse embryos, Nature 292 (1981) 154-156.

[11] Flax J.D., Aurora S., Yang C., Simonin C., Wills A.M., Billinghurst L.L., Jendoubi M., Sidman R.L., Wolfe J.H., Kim S.U., Snyder E.Y., Engraftable human neural stem cells respond to developmental cues, replace neurons, and express foreign genes, Nat. Biotechnol. 16 (1998) 1033-1039.

[12] Fraichard A., Chassande O., Bilbaut G., Dehay C., Savatier P., Samarut J., In vitro differentiation of embryonic stem cells into glial cells and functional neurons, J. Cell Sci. 108 (1995) 3181-3188.

[13] Herrmann B.G., Labeit S., Poustka A., King T.R., Lehrach H., Cloning of the T gene required in mesoderm formation in the mouse, Nature 343 (1990) 617-622. 
[14] Jirmanova L., Pacholikova J., Krejci P., Hampl A., Dvorak P., O-linked carbohydrates are required for FGF-2-mediated proliferation of mouse embryonic cells, Int. J. Dev. Biol. 43 (1999) 555-562.

[15] Martin G.R., Isolation of a pluripotent cell line from early mouse embryos cultured in medium conditioned by teratocarcinoma stem cells, Proc. Natl. Acad. Sci. USA 78 (1981) 7634-7638.

[16] McCaffery P., Drager U.C., Regulation of retinoic acid signaling in the embryonic nervous system: a master differentiation factor, Cytokine Growth Factor Rev. 11 (2000) 233-249.

[17] Mummery C.L., Feyen A., Freund E., Shen S., Characteristics of embryonic stem cell differentiation: a comparison with two embryonal carcinoma cell lines., Cell Differ. Dev. (1990) 195-206

[18] Naegele J.R., Barnstable C.J., A carbohydrate epitope defined by monoclonal antibody VC1.1 is found on N-CAM and other cell adhesion molecules, Brain Res. 559 (1991) 118-129.

[19] O'Shea K.S., Embryonic Stem Cell Models of Development, Anat. Rec. 257 (1999) 32-41.

[20] Okabe S., Forsberg-Nilsson K., Spiro A.C., Segal M., McKay R.D.G., Development of neu- ronal precursor cells and functional postmitotic neurons from embryonic stem cells in vitro, Mech. Dev. 59 (1996) 89-102.

[21] Streatavan D.W., Kruger K., Randomized retinal ganglion cell axon routing at the optic chiasm of GAP-43 deficient mice: association with midline recrossing and lack of normal ipsilateral axon turning, J. Neurosci. 18 (1998) 10502-10513.

[22] Tucker G., Binder L.I., Matus A., Neuronal microtubule-associated proteins in the embryonic avian spinal-cord, J. Comp. Neurol. 271 (1988) 44-55.

[23] Walther C., Gruss P., Pax-6, a murine paired box gene, is expressed in the developing CNS, Development 113 (1991) 1435-1449.

[24] Wiles M.V., Johansson B.M., Analysis of factors controlling primary germ layer formation and early hematopoiesis using embryonic stem cell in vitro differentiation, Leukemia 11 (S3) (1997) $454-456$

[25] Wobus A.M., Rohwedel J., Maltsev V., Hescheler J., In vitro differentiation of embryonic stem cells into cardiomyocytes or skeletal muscle cells is specifically modulated by retinoic acid, Roux’s Arch. Dev. Biol. 204 (1994) 36-45. 